\title{
Immature Granulocytic Sarcoma
}

National Cancer Institute

\section{Source}

National Cancer Institute. Immature Granulocytic Sarcoma. NCI Thesaurus. Code C35818.

A granulocytic sarcoma composed of myeloblasts and promyelocytes (WHO 2001). -2003 